\title{
Violence, respect et sexualité chez les revendeurs de crack portoricains d'East Harlem
}

\section{Philippe Bourgois}

\section{(2) OpenEdition \\ 1 Journals}

\section{Édition électronique}

URL : https://journals.openedition.org/remi/1610

DOI : 10.4000/remi. 1610

ISSN : $1777-5418$

Éditeur

Université de Poitiers

\section{Édition imprimée}

Date de publication : 12 décembre 2002

Pagination : 55-76

ISBN : 2-911627-32-6

ISSN : 0765-0752

\section{Référence électronique}

Philippe Bourgois, « Violence, respect et sexualité chez les revendeurs de crack portoricains d'East Harlem », Revue européenne des migrations internationales [En ligne], vol. 18 - n³ | 2002, mis en ligne le 09 juin 2006, consulté le 15 avril 2022. URL : http://journals.openedition.org/remi/1610 ; DOI : https:// doi.org/10.4000/remi.1610

Ce document a été généré automatiquement le 15 avril 2022.

(c) Université de Poitiers 


\title{
Violence, respect et sexualité chez les revendeurs de crack portoricains d'East Harlem
}

\author{
Philippe Bourgois
}

1 Depuis plusieurs dizaines d'années, le nombre de jeunes noirs et latinos qui ont trouvé la mort dans les ghettos des villes américaines excède largement celui des soldats tombés au feu pendant la Seconde Guerre mondiale. De même, le nombre de Noirs et Latinos placés sous contrôle judiciaire dépasse celui des leurs inscrits à l'université (Mauer, 1992).

2 Les problèmes qu'affrontent les hommes de couleur économiquement faibles alimentent une polémique qui place en général le débat sur un plan moral en dénonçant les carences psychologiques de certains individus psychopathes ou la pathologie sociale de " sous-cultures ${ }^{1}$ ». Situation à laquelle les milieux politiques et les media des années 1990 ont réagi en lançant des croisades exaltant les «valeurs familiales » traditionnelles. Aux États-Unis, il est courant d'analyser la pauvreté et la marginalisation sociale à partir d'une perspective individualiste et ethnocentriste qui rejette la responsabilité sur les victimes. Dans les années d'après-guerre, la sociologie américaine a subi le double traumatisme de la terreur maccarthyste vis-à-vis de l'économie politique et d'un courant "tout américain " qui interprétait l'inégalité sociale en termes d'essentialisme culturel, voire de déterminisme/racisme biologique ${ }^{2}$. Biaisées par des interprétations conservatrices du concept de culture de la pauvreté défini par Oscar Lewis (1966) ou de la dénonciation de la famille noire par Daniel Moynihan (Rainwater et Yancey Éds, 1967), les études consacrées à la violence sont surtout menées par des psychologues et travailleurs sociaux qui s'intéressent à la transmission intergénérationnelle de la maltraitance dans le cadre de thérapie analytique. À de notables exceptions près (Feldman, 1991 ; Sluka, 1990 ; Tausig, 1987) et malgré leur augmentation, les enquêtes anthropologiques consacrées à cette question font l'impasse sur les problèmes du monde industrialisé. C'est ainsi que la «préférence exotique" chère aux anthropologues se nourrit d'enquêtes du type observation 
participante qui sont menées aux marges de l'économie mondiale et qui ne laisse guère de place au dialogue interdisciplinaire (Ferguson, 1988).

3 La sensibilité progressiste et culturaliste des adversaires de l'ethnocentrisme et du "classicisme » inhérente à la plupart des études sur les familles afro-américaines (Burton, 1991 ; Jarret, 1994 ; Stack, 1975)³, ne peut cependant dissimuler la gravité des phénomènes d'abandon paternel et de violence domestique qui caractérisent les milieux pauvres des ghettos américains. Pour comprendre pourquoi, aux États-Unis, un si grand nombre d'hommes économiquement faibles s'entretuent, abandonnent ou maltraitent leur progéniture, il faut resituer le débat au sein du contexte sociostructurel, politique et idéologique où il se pose. Il est clair que la restructuration de l'économie mondiale a considérablement rétréci les possibilités d'emploi des travailleurs les moins qualifiés. On assiste de ce fait à une intensification de la crise matérielle qui à son tour affecte la reproduction du couple et de la famille étendue. En 1994, rompant avec la sécheresse habituelle de ses tableaux statistiques, le Bureau du recensement qualifiait d'«alarmante " l'augmentation de plus de $100 \%$ du taux de pauvreté affectant les familles de jeunes ouvriers au cours des années 1980 et le début des années $1990^{4}$ (New York Times, Mars 31, 1994: A8). Ce climat de détérioration économique et d'hostilité politique à l'égard des marginaux a favorisé la quasidisparition des services sociaux chargés des actifs économiquement faibles (Wacquant, 1995) et entraîné, comme on pouvait s'y attendre, une exacerbation de la ségrégation raciale dans les villes.

L'évolution du patriarcat ouvrier dans east harlem

4 Aux États-Unis, l'aggravation de la marginalisation sociale a considérablement modifié la façon dont les hommes pauvres « accomplissent leur masculinité » (Messerschmidt, 1993). Le marché du travail des débutants et l'économie clandestine condamnent ses participants à la pauvreté, à l'insécurité financière et à des degrés élevés de violence tant personnelle qu'institutionnelle. Le patriarcat ouvrier traditionnel connait une crise matérielle et idéologique prolongée due à l'augmentation constante du nombre d'hommes qui se trouvent dans l'incapacité de reproduire leur «masculinité hégémonique » (Connell, 1987; Messerschmidt, 1993), selon le terme forgé par ces théoriciens en référence à Gramsci (1971). La relocalisation des entreprises en quête de main-d'œuvre bon marché, diminue leurs chances de trouver un emploi stable dans une entreprise syndiquée et donc de bénéficier d'un revenu régulier et d'allocations familiales. Incapables de subvenir aux besoins économiques de la cellule familiale, ces hommes en viennent à perdre la légitimité matérielle sur laquelle se fonde leur exigence de "respect» et sont dans l'incapacité de reproduire les aspirations patriarcales de la génération des grands-parents.

5 Parallèlement à ce mouvement de déstabilisation économique qui touche un patriarcat ouvrier atteint par la restructuration de l'économie mondiale, l'après-guerre a vu un nombre croissant de femmes de toutes origines sociales venir grossir les rangs de la main-d'œuvre ouvrière ${ }^{5}$. Les femmes se sont mobilisées au sein de larges organisations politiques et culturelles ; mouvement dominé, comme l'ont noté les féministes noires, par une forme d'anglo-centrisme " petit-bourgeois » pour qui l'émancipation se définit en termes de droits individuels et de mobilité économique ascendante (Acosta-Belen, 1993 ; Mohanty, 1984 ; Jaggar 1983). Néanmoins et sur le plan idéologique, des secteurs entiers de la société américaine ont vu se disloquer les formes d'organisation patriarcales de la société d'après-guerre. Bien sûr, la réorganisation des structures 
familiales, la restructuration des marchés du travail selon le genre et les modifications des attitudes vis-à-vis de la sexualité n'ont pas suivi une évolution régulière. En outre, les fondements idéologiques et les bases de la domination masculine n'ont pas été touchés. Pour résister à ces changements, c'est souvent par la violence que les hommes tentent de réaffirmer leur contrôle patriarcal sur les femmes et les enfants.

Dans le cas portoricain, les profondes modifications des relations de pouvoir existantes ont été accentuées par un exode urbain massif effectué dans un contexte culturel hostile. Situation encore aggravée par la situation coloniale de Porto Rico vis-à-vis des États-Unis (Melendez et Melendez, Éds., 1993 ; Rivera-Batiz et Santiago, 1994). Peu de peuples ont, dans l'histoire récente, fait l'expérience de tels changements économiques, connu une diaspora aussi massive et souffert d'une marginalisation sociale aussi aiguë, accompagnée d'un mépris culturel patent (Dietz, 1986; Rodrigez, 1989). Une étude approfondie permet donc de mesurer en fonction du genre la disparité des effets provoqués par ces changements historiques. L'étude des Portoricains vivant aux ÉtatsUnis fait apparaître une centralité de la catégorisation de sexe et de genre parallèle à celle de la catégorisation de classe et d'ethnie, le tout au sein d'une " cocotte-minute " sociale chauffée à blanc par les expériences historiques de domination et de résistance.

Le vécu des hommes portoricains de la seconde génération qui participent à l'économie clandestine des ghettos illustre de manière particulièrement impressionnante l'influence de la question du genre sur la souffrance sociale. C'est ainsi que lorsque, entre la fin des années 1980 et le début des années 1990, je me suis installé avec ma famille dans East Harlem, pour mener une enquête sur les revendeurs de crack, la question des relations de pouvoir entre les sexes s'est imposée à moi comme sujet de recherche - et plus précisément le rapport entre la violence personnelle et les luttes masculines pour la dignité (Bourgois, 1995a, 1995b). Cet article s'intéresse donc à la manière dont les hommes portoricains des ghettos, confinés aux marges d'une nation ouvertement hostile à leur culture et que leur force de travail n'intéresse plus, reconstruisent leur conception de la masculinité par le biais de la violence interpersonnelle, du parasitisme économique et de la domination sexuelle. En nombre croissant, ces hommes désespérés et frustrés se sont réfugiés dans une culture protestataire de rue dont le fondement matériel et l'attrait idéologique s'enracinent dans l'économie de la drogue, alternative concrète à leur exclusion de l'économie licite et de la culture anglo-centrée. Les entretiens ethnographiques montreront, je l'espère, que loin d'être les simples jouets de forces structurelles et idéologiques plus vastes, les dealers qui participent à la culture de la rue sont en réalité les agents actifs d'une quête de la dignité - même si cette recherche se manifeste par la violence et l'autodestruction. Ce processus, qui pourrait être « expliqué » comme une psycho-pathologie individuelle, ou rejeté comme une sorte de machisme essentialisme culturel (Paredes, 1971) doit être replacé dans son contexte historique et compris comme l'expression de luttes contradictoires dont les enjeux relèvent du champ du pouvoir et de la signification.

Le contexte historique

8 À l'issue de la Seconde Guerre mondiale, entre un tiers et la moitié de la population de Porto Rico émigra vers les ateliers clandestins new-yorkais (Bose 1986). À ce moment précis, ces emplois manufacturiers quittaient les pays industrialisés. New York devenait le centre logistique des multinationales qui venaient de fermer leurs usines sur place pour les délocaliser à l'étranger où le travail est meilleur marché et certains 
gouvernements plus accommodants (Rodriguez 1989; Sassen 1991). C'est dans ce contexte que sont intervenues les modifications spectaculaires qui ont affecté la structure familiale et les rôles de pouvoir masculins de la seconde, puis de la troisième génération des habitants d'East Harlem. Issus des secteurs les plus pauvres de la société et venus directement de plantations sucrières, fermes familiales marginales ou haciendas caféières en déliquescence, ces immigrants avaient une conception de la masculinité construite autour de réseaux interpersonnels de "respeto" [respect], organisés par catégories complexes d'âge, de genre et de parenté. Les Portoricains se réfèrent souvent à leurs ancêtres campagnards depuis longtemps urbanisés en utilisant le terme de «jibaros ", c'est-à-dire " paysans ». Dans le cadre "traditionnel » classique de la ferme familiale, la valeur du pater familias dépendait du respect que lui manifestaient sa femme et son abondante progéniture. Plusieurs générations plus tard, dans les rues du ghetto, les héritages idéalisés des rangs et marques de prestige jadis attachés à cette tradition familiale rurale et aux hiérarchies personnelles de communautés vivant dans des petites fermes ou des plantations, ont été redéfinis dans un contexte de culture de rue explicitement misogyne et sexuellement agressive. La traditionnelle quête du respect s'est métamorphosée en crainte de l'irrespect. Les chômeurs drogués ou les revendeurs de drogue vivent sans domicile fixe et passent d'une relation sexuelle à l'autre sans jouir de la protection d'une famille ou d'une communauté économiquement viable.

Les souvenirs d'un patriarcat rural refont souvent surface dans les réminiscences d'une enfance idéalisée, évoqués par les mères des revendeurs de crack avec lesquels je me suis lié :

[Madame Ortiz] : «Ce qui me plaisait le plus dans la vie à Porto Rico c'était qu'on conservait nos traditions. Dans mon village, tout le monde était un Oncle, ou une Tante. Quand on croisait quelqu'un de plus âgé, il fallait lui demander sa bénédiction. C'était une marque de respect.

Dans ce temps-là, les enfants étaient respectueux. Mon père était très sévère. Quand quelqu'un venait nous voir, il ne s'adressait à nous que du regard parce que les enfants n'étaient pas supposés se trouver dans la pièce. Il se contentait de nous regarder et ça voulait dire qu'on devait disparaître, aller dans notre chambre. On n'avait pas le droit de se tenir dans la même pièce que les gens plus âgés.

J'ai essayé de transmettre à mes enfants un peu de ce que mon père m'avait luimême enseigné ».

10 Primo, fils de Madame Ortiz et gérant de la crackhouse où je passais beaucoup de mon temps ne peut plus "parler » à ses enfants "avec les yeux", ni attendre qu'ils lui obéissent au doigt et à l'œil. L'oppression domestique qu'exerçait un paysan portoricain était fondée sur sa capacité à travailler et à subvenir aux besoins matériels de sa famille. Il était supposé coordonner leur travail en fonction des cycles agricoles et du travail salarié saisonnier offert par une économie précaire de semi-subsistance rurale. Dans le ghetto new-yorkais, les hordes de jeunes gens exclus du système scolaire et des nouveaux emplois tertiaires qui exigent un minimum d'éducation ont perdu la base matérielle de la prérogative familiale patriarcale du milieu rural portoricain. Les anciens rituels de vénération masculine ne sont plus transmis par le biais du foyer conjugal ou de la famille étendue. Bien au contraire, le chômeur ou trafiquant invective la femme et les enfants qu'il ne parvient plus à faire vivre ni à contrôler de manière effective. Le souvenir de l'ancien pouvoir ancestral le poursuit inlassablement lorsqu'il ressasse un passé jibaro patriarcal qu'il est désormais incapable de reproduire.

La violence domestique 
11 Lorsque que le père de Primo perdit son emploi dans une usine de vêtements, il est devenu «un sale alcoolo" [borrachón sucio] $]^{6}$. Les tout premiers souvenirs que garde Primo de son père le montrent en train de frapper sa mère. Pire encore, tous les hommes qui par la suite partagèrent sa vie offraient à Primo autant de modèles de virilité violente et brutale.

[Primo] : «Quand j'étais petit, j'aimais pas voir quelqu'un avec ma mère. J'aimais aucun de ces hommes, parce j'aimais pas quand ils se battaient et qu'ils la frappaient ; ils devenaient sauvages et ils la mettaient KO.

Y avait un type, quand j'étais encore môme, dans les années 1970, il s'appelait Luis et il sortait avec ma mère. Il lui tapait dessus, juste pour lui faire mal.

Un jour, il s'est déchaîné contre elle, moi je dormais, ça m’a réveillé.

Ma mère a appelé les flics et le salaud, il a pris un couteau. Mon cœur s'est arrêté. C'est la seule fois où j'ai fait quelque chose pour ma mère. Je me suis interposé quand il lui en a flanqué deux ».

Quinze années plus tard, Primo reproduisit ce cycle de brutalité en battant, à son tour, son amie devant ses enfants. En lutte, mais impuissant, contre sa propre incapacité à garder un emploi stable et à gagner le respect de sa famille avec un revenu régulier, il tenait le rôle d'un gigolo vivant aux crochets d'une femme dénommée Candy, une des rares revendeuses du réseau de dealers que je fréquentais. En public, Primo prétendait apprécier «de parasiter » [cacheando] la générosité de Candy et l'amour qu'elle lui prodiguait. En réalité il admit, lors de conversations privées, en avoir été amoureux. Les rôles patriarcaux se trouvaient donc inversés, puisqu'une femme subvenait à ses besoins économiques et qu'il se trouvait contraint de satisfaire ses exigences sexuelles à la demande. Il supporta cette situation quelques mois, mais tenta par la suite de récupérer le respect que lui conférait, pensait-il, sa qualité d'homme par le seul moyen à sa disposition immédiate : la violence physique en public. Des années plus tard, au cours de conversations à la crackhouse, il s'insurgeait contre le fait que sa conception de la masculinité ait été mise à mal [dissed] parce que Candy avait violé les règles en renversant les rôles domestiques :

[Primo] : «Cette femme me traitait grave. Putain, elle se faisait des masses de fric. Cette garce, elle s'en faisait encore plus que j'croyais.

Elle valait rien, cette pute. On aurait dit que ses mômes, ils avaient pas de mère. Ils s'occupaient de tout tous seuls. Sa petite, Lilian, elle avait même pas un an. Merde! C'était le grand qui faisait la mère. C'était lui qui lui changeait les couches.

Et quelquefois, j'me sentais mal, et pas à l'aise, alors, c'était moi qui le faisais. J'étais là, à changer les couches, alors qu'elle, elle était même pas là !».

Pire encore, Candy inversait les rôles du machisme patriarcal :

[Primo]: «En plus, tu vois, style, quand elle revenait à la maison, elle était de mauvais poil, ou dans le genre, mais moi, je voulais pas d'embrouilles ».

13 Après avoir quitté le foyer de Candy à la suite d'une violente défonce, Primo reprit son activité de revendeur de crack à plein temps. Quand la soirée était calme, nous avions droit, César le guetteur de la crackhouse et moi, au récit détaillé de la dernière bagarre qui avait mis un terme à la relation de dépendance économique et sexuelle qu'il entretenait avec Candy. Tout avait commencé quand Primo avait refusé de lui faire l'amour, Candy l'ayant alors accusé d'avoir des petites copines. Primo semblait presque utiliser les entretiens enregistrés à la crackhouse comme une thérapie destinée à résoudre la confusion générée par le viol des tabous de genre lorsqu'il vivait aux crochets de Candy. Il avait également besoin de se créer un lien avec ses copains de la crackhouse en se targuant d'avoir triomphé d'une femme de mauvaise conduite. Le 
récit atteignait son apogée au moment où il avait désarmé Candy pour la battre plus rudement devant ses enfants :

[Primo] : «Dès qu'elle a baissé son arme - je ne me rappelle pas bien où elle l'a posée, mais j'ai vu qu'elle enlevait le chargeur - j'ai gueulé "Sale pute" (brandissant les deux poings). Et je te l'ai bousillée.

J'étais bourré, mon vieux. Je gueulais : "Allez, sale garce. Je jouerai plus jamais avec toi !"

[Philippe] : Et les gosses?

[Primo]: Ils étaient là, dans la pièce, ils étaient énervés, je suppose qu'ils pleuraient...

Je vais te dire, les enfants, ils savaient que leur mère avait tort, mais comme moi je la tapais, ils avaient envie de me sauter dessus. Quand j'ai vu leurs visages, j'ai compris qu'il fallait que je me prépare. J'étais prêt à, comment dire, à parer leurs coups ".

Des hommes vulnérables

La manière dont Primo s'efforce de légitimer sa propre violence envers Candy contraste avec la condamnation qu'il porte sur la violence exercée par son père contre sa mère et ses sœurs. Il juge ouvertement que son père est un raté, il n'a jamais pu lui témoigner le respect dû par un enfant de jibaro à son père :

[Primo] : « Aujourd'hui c'est un malade. Il est diabétique. Il est alcoolique et quand il boit, il devient violent. C'est comme qui dirait une lopette, alors, pourquoi je le verrais, moi ? C'est pour ça que ma mère a été obligée de lui crier : "Allez, ouste" [avec un grand sourire inattendu, il fait le geste de l'arbitre expulsant un joueur]. Et moi je voyais mon père tous les quinze jours après leur séparation - vu qu'ils n'ont jamais divorcé. Et il était pas recto, c'est tout. Toujours une bière à la main. Toujours bourré, toujours en train de beugler.

Nous on était mômes. On se disait : "Va te faire foutre, moi j'm'en balance". Il nous achetait des bonbons. Et puis, après, il venait me demander: "Ta mère, elle a quelqu'un?". Je me rappelle pas ce que je lui répondais mais je devais lui dire "oui", ou n'importe quoi. Il était bourré et il était con.

Peut-être qu'il a eu des regrets. Et il aurait pu vivre mieux que ça, financièrement parlant. Je me rappelle plus trop bien. Et puis voilà qu'il s'effondrait, il avait la tremblote. Moi, j'avais la haine ».

15 L'image de ce père accablé par une crise de jalousie au moment où son fils lui apprend que son épouse abandonnée a un nouvel amant illustre ce symptôme psychosomatique portoricain: la «crise de nerfs» [ataque de nervios] (Lewis-Fernandez, 1992; Guarnaccia et al., 1989). Ces « ataques » provoquées par la jalousie, la maltraitance ou le manque d'amour, sont habituellement le fait des femmes. Que le père de Primo ait pu passer par cette expression typiquement féminine du désespoir et de la détresse devant ses enfants et amis proches illustre le sentiment d'impuissance masculine qu'il doit avoir éprouvé aux États-Unis en tant que migrant marqué par l'échec. Comme mécaniquement, dans le style macho raté, il se remettait en s'attaquant à la femme vulnérable la plus proche, à qui il ne commandait plus le respect depuis longtemps.

$16 \mathrm{Au}$ bout du compte, Primo finit par convenir de son propre échec en tant qu'homme. Phénomène qu'il désigne en termes biologiques comme un "truc mâle ». La manière émouvante dont il décrit comment plusieurs générations d'hommes de sa lignée directe se sont fait broyer, laisse apparaître la spécificité de la marginalisation sociale qui affecte la diaspora portoricaine :

[Primo]: «Je te le dis Philippe, il faut que je me flingue. Parce que, comme je le disais à ma mère, dans ma famille, c'est comme ça que ça se passe, tous les hommes, ils sont pétés. 
Le frère aîné de ma mère est pété. Il parle tout seul devant la fenêtre.

L'autre frère de ma mère - un autre oncle à moi - il fait que marcher comme un zombie et il regarde personne. Je suis son neveu et son filleul, les deux. Il écrit des gribouillis, on dirait de la sténo, mais c'est pas de la putain de sténo. Il écrit dans son carnet, il gribouille, il fait des barbouillis dans son carnet. Mais ce mec-là, il a son boulot. Il garde son boulot. Il garde sa place, mais il a plus sa tête.

T'as qu'à le regarder marcher dans la rue, on dirait un clodo. Il avance tout droit, les yeux baissés. Il est fêlé.

Je me rappelle quand il était pas fêlé. Il est allé à Porto Rico avec ma mère, quand il était pas fêlé.

Tu sais quoi, moi je dis à ma mère que pour moi, tous les gens de ma famille, les mecs, je veux dire, ils vont tous péter les plombs un de ces quatre. Je m'imagine plus tard, moi aussi et je me dis que je serai fêlé.

Mais il se trouve que par contre, mon grand-père, lui, il était pas fêlé. Mon grandpère est décédé, mais il était pas félé, il est mort, c'est tout ». dans l'économie parallèle ni n'ont même participé à la violence de la culture de rue. Soit elles travaillaient à plein temps dans le secteur tertiaire (gérante de restaurant McDonald's, serveuse dans des boutiques de vêtements et aide-soignante à l'hôpital municipal d'East Harlem), soit elles s'étaient mariées et élevaient leurs enfants. Primo était fier de leur réussite, ce qui illustre bien non seulement à quel point les rôles des femmes sont rigidement définis mais aussi, encore une fois, à quel point, dans le contexte de pauvreté du Barrio, elles évoluent différemment :

[Primo] : Tu sais, ma mère, elle est bien. Elle a élevé trois filles qui ont bien réussi.

Elles se sont mariées un peu tôt, et tout, mais rien à voir avec la drogue dans la rue.

Elles savent le bien et le mal.

Mes sœurs, elles sont pas violentes. Elles sont pas dans la rue - pas de ça. Stabilité conjugale et emploi légal

Contrairement à ce que pouvait laisser supposer leur comportement souvent bravache et leurs diatribes ouvertement misogynes, la plupart des 24 revendeurs de crack avec lesquels je m'étais lié avouaient aspirer à fonder une famille "bourgeoise " idéale de type nucléaire. Ils avaient en fait vécu dans des foyers stables avec les mères de leurs enfants pendant des périodes assez longues qui correspondaient à celles où ils occupaient des emplois légaux et stables. Au cours de nos entretiens, l'interrelation complexe entre chômage, pathologie individuelle, instabilité familiale et fragilité structurelle sur le marché du travail intervient de façon récurrente. Le récit qui va suivre rend compte du lien entre travail et prise de drogue dans la vie de Primo, il illustre l'interaction entre personnalité et structure sociale dans la construction des subjectivités masculines? ${ }^{7}$.

[Primo] : «J'avais dix-neuf ans quand j'ai eu mon môme. Il est né en 1983, le vingt et quelque chose de mai. On était ados, on sortait ensemble, Sandra et moi. J'avais trouvé du boulot et on se fréquentait. On a dégotté une piaule, moi je gagnais bien. J'étais un bon nèg', mon vieux. Tout le fric que je faisais il passait dans la CB. C'était mon truc. Elle est tombée enceinte. Nous, on le voulait pas vraiment. Mais moi je lui ai dit: "Je suis aussi responsable que toi, alors si tu veux le garder, j'assume". Alors elle l'a gardé. C'est dommage. Mais bon, ça va.

Et je vendais pas de came, ni rien. J'étais un gentil petit gars. J'avais du fric à la banque, j'avais de la thune à la maison. Sandra, elle n'a jamais souffert. Elle était balaise, elle était grosse et elle était enceinte.

Quand Pepito est né, moi je travaillais à U.S Litho. J'étais un bon nèg'. J'avais des bons horaires. Je travaillais de seize heures à minuit.

Revue européenne des migrations internationales, vol. 18 - $n^{\circ} 3$ | 2002 
Je bossais dur. Je faisais des heures supplémentaires. Le boulot qu'ils me donnaient, je le faisais. Je voulais ramener du fric à la maison.

C'est comme ça que j'ai arrêté de sniffer [de la cocaïne]; un jour mon fils a voulu jouer avec moi. J'étais dans le rocking-chair et moi j'avais pas envie de jouer avec lui, j'étais genre "foutez-moi-la-paix", putain de merde. Et puis j'ai réfléchi et je m'en suis rendu compte. J'ai remarqué que mon fils grandissait. En plus, un jour, j'ai eu des saignements.

J'ai dit: "Non, ça, c'est pas possible. C'est pas moi, ça". Parce que je suis toujours gentil avec mes gosses, je leur chante des chansons, des petites chansons que j'ai appris à l'école, quand j'étais môme.

Alors je m'asseyais dans un rocking-chair, je lui lisais son alphabet et les chiffres, juste pour lui occuper la tête. Les gosses, il faut leur lire des trucs, quand ils sont petits, même quand ils ont que dans les neuf mois, comme ça ils ont toujours des machins qui se stockent dans leur cerveau.

Et puis après, ils ont changé mes horaires, de deux heures à dix heures du matin. Alors j'ai dit "C'est pas possible, un horaire pareil, j'ai une famille, moi".

Je m'endormais au boulot. Vu que j'avais mon fils. Et cette nana, Sandra, la mère de mon fils, elle avait trouvé un boulot au noir. Et quand elle partait, moi je rentrais et mon fils me grimpait dessus. Il avait envie de jouer. Il avait fini de dormir, tu comprends, alors moi, impossible de dormir.

Et c'est là que j'ai commencé à déconner. J'ai commencé à fumer des "woolas" [cigarettes de marijuana mélangée avec du crack], et je buvais un peu. J'étais debout toute la journée, alors après, j'avais pas envie d'aller bosser.

Tu piges, c'était comme si en rentrant du boulot je savais pas si je devais aller me coucher tout de suite ou traîner un peu pour me coucher plus tard. Et mon fils, il était là, frais comme un gardon. Il avait deux ans, il avait envie de jouer.

Alors ils m'ont vidé parce que je m'endormais au boulot. Ils m'ont dit : “faut qu'on te laisse partir parce que t'as une famille et on sait que tu veux être avec eux, à cause de ton horaire, mais nous, on peut pas te remettre dans l'équipe de jour. On a besoin de quelqu'un la nuit et toi, t'as pas l'air de t'y faire".

Ils viraient tout le monde, il cherchaient juste un motif. À mon avis, leurs affaires elles tournaient pas rond.

Après ça, je me suis tiré et j'ai fumé du crack.

Primo ressentait vivement son échec en tant qu'homme et en tant que père :

[Primo] : «Tu sais quoi, Philippe? Mon fils, il a six ans maintenant. Ça me fout le cafard quand je pense à toute cette merde... C'est du style que je suis pas là pour lui. Exactement comme mon père, il était pas là pour moi.

Et mon fils, il aimait bien être avec moi. À un moment j'étais toujours en train de bricoler quelque chose dans l'appartement. Alors le gosse, il attrapait mes outils, et il donnait des coups de marteau sur n'importe quoi, comme ça, il me regardait et il faisait pareil. Ça me plaisait ça, putain.

C'est pour ça que j'ai pleuré beaucoup la première fois que j'ai quitté mon fils. C'était à peine deux mois après qu'ils m'ont renvoyé de U.S Litho.

J'allais dans la salle de bains et je chialais comme une mauviette. Parce que je savais que j'allais bientôt me tirer et que ça, ça voulait dire une chose : plus de gosse.

Mais tu sais quoi, mec. Moi je crois... je crois que quand t'es avec quelqu'un et que t'as un gamin, faut que tu fasses le max pour en profiter, que t'y arrives ou pas. Faut que tu t'engages, comme pour un truc de famille, dans l'ancien temps. »

[Philippe] : Non, mais, bordel, de quoi tu parles?

[Primo]: On dirait tout le temps que je suis qu'un enculé, quand je dis des choses comme ça, parce que je m'occupe pas de mon gosse, mais c'est parce que je... en ce moment, je m'en occupe pas mais...

En fait, tu te rappelles, le week-end dernier, à un moment quand on a parlé de la dernière fois qu'on avait pleuré, et ben, pour moi c'était cette fois-là, le week-end dernier. J'ai pensé à mon petit nèg'. Normalement j'aurais dû le prendre pour le week-end mais j'ai appelé trop tard. J'ai merdé. Un vrai merdier. 
[D'un ton plus vif] En fait, je me rappelle mon père qui racontait à ma mère qu'il pleurait parce que je lui manquais, parce que j'étais son fils unique.

Mais là, je suis mal parce que dans quelques jours c'est l'anniversaire de Papito et j'aurai rien à lui offrir. Parce que j'ai pas le fric.

[Philippe] : Tu préfèrerais pas avoir les 25 dollars que tu as dépensé pour la blanche [héroïne] et le perico [la cocaïne] pour lui acheter un cadeau d'anniversaire ?

[Primo, en sniffant un sachet d'héroïne] Ben... Je vais lui acheter un cadeau.

J'l'adore ce petit nèg' ".

L'occupation d'un emploi licite par les deux conjoints est décisive pour que les hommes puissent commencer à définir la masculinité en termes de partage de la reproduction des enfants, sur le plan matériel comme émotionnel. Le problème est qu'à New York, la plupart des emplois licites que peuvent occuper les jeunes sortis du système scolaire sont non seulement mal rémunérés mais également considérés comme féminisants. Ceci parce que le secteur tertiaire et en particulier l'administration des industries de la finance, du logement et des assurances ont connu leur plus forte croissance depuis les années 1970. La majorité des agents de surveillance des niveaux inférieurs du secteur tertiaire sont des femmes et la culture de rue fustige les hommes qui se trouvent en position d'infériorité dans le rapport hommes/femmes. Il est intéressant de noter qu'en citant leurs mauvais souvenirs d'irrespect au travail, de nombreux trafiquants de crack font référence à leurs supérieures dans un langage explicitement sexiste. Ils s'en prennent souvent à leur apparence physique et manifestent leur rejet en utilisant l'argot des rues et des jurons sexualisés. Ils décrivent souvent ces femmes qui leur sont hiérarchiquement supérieures comme ayant des organes sexuels mâles et ils se décrivent eux-mêmes et les autres hommes de leur entourage professionnels comme des efféminés.

[César]: «Je suis resté dans la salle du courrier quelque chose comme huit mois, dans cette agence de pub qui travaille avec ces merdes de produits pharmaceutiques. Ils me faisaient confiance.

Mais ma patronne, elle avait des préjugés. Une vraie salope, cette pétasse. Une blanche. Elle me faisait faire des tas de merdes, cette sale grosse mochetingue, et moi, j'étais comme une vraie mauviette.

Ça me plaisait pas, mais je continuais à travailler parce que putain, tu veux pas bousiller une relation comme ça. Alors t'es qu'une lopette.

Je pouvais pas la saquer, cette cheftaine. Elle était franchement mauvaise, cette pute. Elle prenait son pied en virant les gens. Ça se voyait sur sa tête, mon vieux. Elle a fait chialer le type qui bossait avec moi-même qu'il l'a suppliée de le reprendre ».

21 Les emplois tertiaires d'une économie post-industrielle marquée par la globalisation et les changements technologiques n'offrent que de maigres chances aux jeunes gens des ghettos sortis du système scolaire. Non seulement ils éprouvent des difficultés à accepter d'être commandés par des femmes, mais ils considèrent souvent qu'il est carrément émasculant de devoir courir chercher du café pour leurs supérieur(e)s hiérarchiques et pis encore, d'avoir à le faire avec un grand sourire. En conséquence, même lorsqu'ils conservent leurs postes de coursiers, d'opérateurs de photocopies ou de grouillots, ils ont tendance à se faire une réputation de mauvais "esprits " ce qui limite encore davantage leurs chances de monter dans la hiérarchie administrative.

Primo n'a jamais acheté de cadeau d'anniversaire à Papito, son fils de sept ans. Il n'est d'ailleurs même pas allé le voir cette semaine-là. Par coïncidence, à la même période dans la véranda de mon bâtiment, Manny et Angel, mes petits voisins me fournirent des aperçus de l'autre face du fossé générationnel père-fils. L'œil étincelant, Angel me dit 
avec fierté : « Moi, ce week-end, je vais voir mon père ». Son petit frère Manny répondit immédiatement sur un ton terne et triste : " Moi je verrai pas le mien, il est en prison ». Comme dans un film, quelques instants plus tard, nous entendîmes les hurlements de plaisirs du fils de mon voisin, un autre petit garçon âgé de trois ans surnommé Papo. Un jeune homme de vingt ans, l'air fanfaron et presque embarrassé embrassait le petit garçon, en marmonnant affectueusement «Ay mi hijo " [oh, mon fils] sous le regard apparemment impassible de la mère. Le père de Papo, incarcéré pour trafic de drogue, bénéficiait ce jour-là d'un après-midi de liberté dans le cadre d'une mesure de réhabilitation par le travail. Une heure plus tard environ, le petit Papo repoussait des cris stridents, mais d'angoisse cette fois. Son père devait se dépêcher de rentrer pointer à la prison avant le crépuscule. Le gardien de l'immeuble m'expliqua plus tard que le père de Papo avait cambriolé l'appartement de la mère de l'enfant deux ans et demi auparavant, alors que Papo n'avait que six mois. Il savait que l'appartement était vide, puisqu'à cette heure-là il était supposé être au parc avec son fils. Sa nouvelle copine faisait le guet pendant qu'il dérobait le magnétoscope et la télévision.

Investir dans le multipartenariat sexuel

La conquête sexuelle et la multiplicité des partenaires représentent un autre lieu de définition de la dignité masculine propre à la culture de rue. Il est lié au besoin de rendre positif le "parasitisme » économique exercé aux dépens des mères, amantes, femmes et enfants qu'on ne peut plus soutenir financièrement (Anderson, 1999; De la Cancela, 1986). César, le guetteur de Primo, était le plus vif à célébrer son incapacité à entretenir une famille: incapable de reproduire les aspirations patriarcales de la génération de ses grands-parents - dans le contexte d'une famille nucléaire contraignante et d'une communauté de parentèle étendue -, il concentrait ses mâles énergies en un machisme à sens unique, collectionnant les aventures et travaillant activement à exagérer ses exploits sexuels et à se faire passer pour un sans-cœur.

[César]: « Nous, on est exactement comme ces tortues de mer vertes qu'il y a aux Galapagos. Elles sortent de leurs carapaces, elles courent vers la mer et elles ne savent jamais qui c'était leurs parents.

Elles vivent leur vie. Ensuite, elles baisent quelqu'un ou elles se font sauter. Elles ont des mômes et elles les voient jamais.

Moi je me sens pas coupable vis-à-vis des mômes que j'ai faits à droite ou à gauche parce que moi, Felipe j'ai pas de cœur. Moi je peux baiser n'importe qui, n'importe quand. Et en plus, ces salopes, de nos jours elle sont toutes pétées ».

Primo était plus fonctionnel dans sa sexualisation du pouvoir masculin, faisant porter l'accent sur un contrôle économique et affectif discret des femmes avec qui il sortait. Un soir, tard après son boulot à la crackhouse, je l'accompagnai boire de l'alcool de contrebande dans un club du coin. En avalant ses lampées d'un air rigolard, Primo fit signe à trois joueuses de billard qui se trouvaient un peu plus loin. Imitant un accent campagnard portoricain démodé, Primo dit en espagnol: «Regarde comme elles s'entendent bien, mes femmes » [Mira como mis mujeres se llevan bién].

À l'époque, le juge de tutelle pressait Primo de trouver un emploi. Un juge de la section des narcotiques de New York l'avait condamné à une peine de deux à quatre ans avec sursis pour avoir vendu du crack à un agent en civil. Une des conditions de cette liberté surveillée était qu'il trouve un emploi licite. Pendant qu'il intriguait pour savoir avec laquelle de ses femmes il allait passer la nuit, je lui rappelai à dessein que s'il ne voulait pas aller en prison, il fallait qu'il appelle l'agence pour l'emploi tôt le lendemain matin. 
Primo changea simplement de sujet et se réfugia dans le récit de ses conquêtes sexuelles.

[Primo, d'un air sombre]: «Oh merde, Felipe, t'as raison! Mais tu sais quoi? [Gloussant] De toutes ces pétasses qui traînent ici, c'est celle-là que je me choisis [désignant Maria].

[À nouveau l'air sombre] : Ça m'est déjà arrivé. Un jour, devant la salle de jeux, j'ai vu une masse de nénettes dans un coin. [Gloussant à nouveau] Comme un troupeau de filles qui discutent. Et quand j'ai regardé, j'ai dit : "Mais bon Dieu, je me les suis toutes faites, celles-là". Y avait Sandra, mon ex-femme, Candy, Maria, Jaycee, et une autre, je crois, mais je me souviens plus d'elle.

[Philippe] : Tu t'es senti comment? Bien?

[Primo]: Non. Je m'suis senti bizarre. [Remarquant César qui tendait l'oreille à cause de la musique tonitruante] Non, d'abord je m'suis senti bien, et puis après, je m'suis senti bizarre.

Je te le dis, Felipe, moi j'ai une bite en or. Tous mes cousins sont comme ça. [Donnant cinq tapes à César]. On a tous des bites en or ».

Le viol collectif

Vers la fin de mon séjour à East Harlem, plusieurs des trafiquants de crack avec qui je m'étais le plus lié m'avouèrent avoir participé à des viols collectifs quand ils étaient adolescents. Aux États-Unis, il est rare que les enquêtes sur les bandes menées par des criminologues et des sociologues fassent mention du viol (Campbell, 1991 ; Huff, 1990). Celui-ci est en général présenté comme un rituel initiatique, mais on note parfois que cet acte violent sert à lier les garçons dans un érotisme homosexuel et misogyne (Bourgois, 1994a : chapitre 5), la participation aux viols collectifs est pour les jeunes garçons une façon d'affirmer leur virilité.

Toutes ces dimensions ritualisées - l'atteinte de la majorité, l'attachement mutuel et le rituel initiatique - s'appliquent aux récits des viols adolescents collectifs rapportés par les revendeurs de crack. Primo, par exemple, n'éprouvait aucune excitation sexuelle lorsque ses camarades commencèrent à violer une des filles appartenant à leur réseau. Il se sentait humilié et exclu lorsqu'ils le renvoyaient chez lui au prétexte qu'il était « trop petit » pour participer à leurs exactions sexuelles.

[Primo] : « À cette époque-là, j’étais plus jeune. J'arrivais pas à bander. Il m'obéissait pas, le petit salaud. J'étais pas dans le coup. Je pige pas ça.

Alors, ils montaient avec une fille et, tu penses bien qu'ils savaient déjà que j'allais pas être dans le coup. Alors ils me demandaient : [d'un ton hostile] "Alors, qu'esse tu fous, mec ? Tu rentres à la baraque, ou quoi ?"

Alors, putain, la seule chose que je pouvais faire c'était de me tirer. "À demain, les gars", ou alors j'attendais en bas, au bar, ou style.

C'est en devenant complice de viols collectifs que Primo se fit accepter par les garçons plus âgés qu'il admirait.

[Primo] : J'étais pas vraiment dans le coup mais moi aussi, je me déchainais parce que la pétasse, il fallait bien qu'elle y passe. Et quelquefois, c'était moi qui faisais le mariole avec une batte ou style, comme ça elle était bien obligée de rester dans la pièce avec celui qui était là.

Quelquefois, les grands, ils faisaient les seigneurs un certain temps avec la fille, mais une fois qu'ils l'avaient baisée, elle était foutue. C'était style: Vlan, et vlan [comme s'il donnait des gifles], elle se faisait avoir par style: "T'es à moi, maintenant, pétasse".

Ça c'était il y a longtemps. Personne fait plus ça aujourd'hui. La baise est devenue trop facile ».

Outre l'accent porté à cette dimension rituelle des viols collectifs dans les milieux adolescents et sa fréquence particulière au sein des bandes de garçons, il est important 
de situer le viol public dans le cadre où j'ai placé la violence domestique. Par le viol collectif, les mâles donnent une dimension publique à leur tentative de réaffirmation des relations de pouvoir patriarcales anachroniques qui étaient l'apanage des générations précédentes et qui ont été affaiblies par la modification des rapports de pouvoir de sexe. Le fait que les filles gagnent en autonomie dans les milieux dominés par les mâles incite ceux-ci à se débattre vigoureusement. Ils légitiment leur violence sexuelle contre les jeunes adolescentes au prétexte de «leur infliger une leçon ». En fait, dans leur argot, le viol collectif se dit « lui faire passer un train dessus » [to run a train on a bitch] qui devient parfois «dresser une chienne» [training a bitch ${ }^{8}$ comme si la personne agressée était un chien à qui on donne une leçon ou à qui on enseigne un nouveau tour. Primo, qui avait conscience de l'horreur et de la colère que m'inspiraient ses récits de viols collectifs, argumentait souvent avec moi pour me faire comprendre comme à toute personne qui lirait la transcription de ces entretiens - la dichotomie entre les victimes de viols collectifs qui étaient dignes de respect et celles qui ne l'étaient pas :

[Primo] : « On va le dire comme ça, Felipe, ces pétasses elles étaient jeunes, idiotes et super chaudes. Si elles trainaient trop alors qu'elles se rendaient compte qu'on était dingues et si elles continuaient à traîner, alors, nous on savait qu'on pouvait les prendre.

[Philippe] : C'est bien de la merde, ce que tu dis. Bande d'enculés, vous étiez rien qu'une bande de pervers, oui.

[Primo, frustré] : Non, mais regarde leur attitude, si elles traînaient trop longtemps, crois-moi, c'est qu'elles savaient ce qui se passait. Si la meuf elle traîne, elle va se faire sauter. Je veux dire, ces pétasses, elles arrêtaient pas de glander, de traîner. Elles revenaient au bar tous les jours alors, on le savait qu'elles voulaient se faire sauter.

Ils emmenaient la meuf à part, parce qu'on avait sa confiance [confianza]. C'était pas difficile, après, de l'obliger à se faire sauter par chacun à son tour.

En plus, elle se faisait taper ou style, si elle refusait.

[Philippe] : C'est du viol, ça mon vieux. Tu piges pas ça ?

[Primo]: Moi, je veux dire dans mon souvenir, (s'adressant directement au magnétophone), c'est que j'étais qu'une putain de môme. Je voyais ça comme qui dirait, que si y en avait qui revenaient pas traîner au club, c'est qu'elles avaient été traumatisées et que ça allait rester enfoui dans leur vie, pour toujours, et qu'elles reviendraient jamais traîner par là. Au lieu de revenir, elles rentraient chez elles se planquer [me jetant un regard défensif] pour garder leur sombre secret jusqu'à la fin de leur vie. Moi, quelquefois, je les plaignais, aussi.

Mais y avait des pétasses qu'étaient plus arrangeantes et elles revenaient glander dans le coin. Parce qu'à mon avis c'était comme si elles étaient dans la rue et qu'une fois passé leur premier truc de merde, après, il leur restait plus qu'à se faire foutre. [Philippe, l'interrompant]: Arrête, vieux, déconne pas. Personne n'aime ça, une pareille saloperie.

[Primo, d'une voix lente] : Eh ben... C'était leur choix, Felipe. Ce que je veux dire c'est que, la première fois, peut-être qu'elles étaient pas d'accord. Quelquefois, elles avaient les larmes aux yeux. Elles voulaient pas être prises de force.

[César, se moquant de la gêne manifestée par Primo et de ma colère, m'arrache le magnétophone]: Mais oui, elles étaient forcées, mais ça leur plaisait. Et elles en redemandaient, parce qu'elles étaient d'accord. Elles s'habituaient à ce fait-là, c'est tout : "T'es à nous, maintenant pétasse".

[Philippe] : Mais bande d'enculés, vous êtes malades [des coups de feu retentissent tout près, puis un bruit de course].

[Primo, se précipitant vers la porte pour voir d'où proviennent ces bruits] : Non! Faut que tu piges, Felipe, même quand elles disent non, elles aiment ça ». 
En fin de compte, la violence, contre les femmes, orchestrée par les modèles de référence de Primo en dit long sur l'intériorisation de leur propre dévalorisation.

[Primo]: «On discutait entre nous, on se disait que ces filles, elles se faisaient sauter parce qu'elles voulaient traîner avec nous.

Mais putain, qu'est ce qu'on avait à leur proposer? Rien. Alors, on se posait des questions.

[César] : Nous on en foutait pas une! Quelles connes, de sortir avec des nèg' comme nous".

Responsabilité individuelle et victimisation structurelle sociale expérience d'oppression et de marginalisation sociale évoque certaines images négatives qui risquent de renforcer certains stéréotypes sociaux et raciaux. Un débat ouvert et non censuré sur le sujet de la masculinité dans la culture de rue peut se transformer en lieu d'humiliation pour les plus pauvres et les plus démunis. Les critiques culturalistes chicanos ont fait remarquer, il y a longtemps déjà, à quel point les perceptions anglo-saxonnes du machisme latino sont le reflet de préjugés historiques bien enracinés (Paredes, 1971)9. Plus grave encore, les propos ethnocentriques sont si inconsciemment incrustés dans le «bon sens " général que les tableaux de misère et de brutalité sociales extrêmes comme ceux qui sont présentés ici sont interprétés comme la représentation culturelle d'une communauté ethnique donnée - en l'occurrence, les immigrés portoricains aux États-Unis. Il est évident que cette attitude, qui relève du " déballage de linge sale ", est en totale contradiction avec les arguments historiques et théoriques présentés dans cet article et qui s'opposent aux explications culturelles essentialistes des comportements humains. Il en est de même pour toute interprétation accusant les femmes d'avoir - dans la rue ou chez elles suscité la violence masculine qu'elles subissent en revendiquant l'extension de leurs droits. Ce genre d'interprétation tenant la victime pour responsable de son sort a non seulement pour effet de magnifier la pérennité du statu quo patriarcal antérieur, mais de surcroît d'individualiser à l'excès la transformation macrostructurelle qui, sur tout le globe et à long terme, est en train de s'accomplir dans les relations entre les sexes. Comme cela s'est toujours passé historiquement, pour tous les changements politiques majeurs affectant des groupes antagonistes, le processus complexe par lequel les femmes se définissent un nouvel espace public abonde en conséquences contradictoires et provoque bien des souffrances ${ }^{10}$. Comme je l'ai noté au début de cet article, le fait que les statu quo fondamentaux qui permettent l'exercice de la domination masculine n'aient quant à eux subi aucune modification rend la situation encore plus critique. Bien des féministes l'ont souligné depuis longtemps : au cours des dernières décennies, la plupart des luttes et réalisations des femmes se sont définies dans le cadre de droits individuels qui sont en fin de compte largement calqués sur les modèles patriarcaux bourgeois d'" accès à l'autonomie » [« empowerment »] (Butler et Scott, 1992; Jaggar, 1983).

Sur un autre plan théorique, la compréhension des expériences d'oppression et de résistance - masculines ou non - souffre de la médiocrité des analyses concernant le rapport personnalité / société, ainsi que le note Jefferson (1994). Les relations entre « agissement » [agency] et structure sont en général présentées en termes dualistes - si tant est qu'elles soient d'ailleurs traitées (Giddens, 1991). La sexualité, l'organisation familiale, les rapports sociaux de sexe et la construction culturelle de l'intimité sont des questions cruciales qui affectent les menus détails de notre vie quotidienne. À bien des égards, ils restent des sujets tabous pour les ethnographes qui ont pour mandat 
inconscient de présenter des images positives des gens qu'ils étudient et avec lesquels ils vivent. Les chercheurs qui procèdent à des enquêtes quantitatives n'ont jamais eu accès à ces dimensions complexes de la vie quotidienne, puisque la plupart des gens dissimulent leurs expériences intimes de violence, de sexualité, de toxicomanie, etc..., même à leurs amis les plus proches - et d'autant plus à l'enquêteur.

Il n'existe pas de solution définitive aux problèmes complexes nés de cette décision de présenter des entretiens ethnographiques intimes transcendant les frontières de classe et de race. Les hommes dont il est question dans cet article se comportent souvent de manière cruelle et violente, non seulement vis-à-vis des femmes et des enfants qui partagent leur vie, mais également vis-à-vis d'eux-mêmes. En dépit du risque que j'ai pris en présentant ce matériau difficile, je pense que si j'ai échoué dans mon entreprise - en particulier dans les aspects les plus pénibles de la misogynie et de la violence sexuelle dans la culture de rue et les actes individuels, - je me rendrai complice de ce statu quo sexiste. Si tous les revendeurs de crack sont des victimes d'un point de vue structurel social, ils sont également les agents de leur propre autodestruction au quotidien. Ils provoquent des ravages sur ceux qui leur sont chers et audelà, sur l'ensemble de la communauté. Bien sûr, d'un point de vue analytique, les formes particulièrement brutales que la masculinité a revêtu dans les rues des ghettos américains - ou les taux de criminalité, d'homicide et d'incarcération dépassent ceux de l'ensemble du monde industrialisé - sont finalement la manifestation, non seulement du modèle économico-politique américain, mais bien plutôt des carences fondamentales dont souffrent, en matière de droits de l'homme, les milieux défavorisés et marginalisés aux États-Unis - en particulier dans le domaine de la liberté individuelle, de l'accès aux soins, au logement, à l'éducation et à la sécurité publique des habitants des ghettos. Autrefois fondées sur une structure de vie rurale, les subjectivités masculines patriarcales se sont polarisées sur l'exacerbation de la violence, la maltraitance sexuelle généralisée et le parasitisme économique vécu au grand jour. Phénomènes qui ne sont que la manifestation symptomatique de ces inégalités fondamentales au plan politique et culturel. Derrière les conversations les plus crues qui sont évoquées dans ce texte, se cache, d'une part, la crise massive des secteurs public et privé qui a secoué la plupart des ghettos américains depuis la Seconde Guerre mondiale, et de l'autre, l'idéologie d'apartheid de facto qui légitime un «bon sens " généralisé prêt à tolérer l'élévation des seuils d'appauvrissement dans la population travailleuse la plus démunie.

\section{BIBLIOGRAPHIE}

ACOSTA-BELEN Edna (1993) «Defining a Common Ground : The Theoretical Meeting of Women's, Ethnic, and Area Studies ", in Edna Acosta-Belen and Christine E. Bose, eds, Researching Women in Latin America and the Caribbean, 175-186, Boulder, CO : Westview Press.

ANDERSON Elijah (1989, January 1) « Sex Codes and Family Life Among Poor Inner-City Youths », The Annals of the American Academy of Political and Social Science, 50, pp. 59-78. 
BACA ZINN Maxine (1989) «Family, Race, and Poverty in the Eighties ». Signs : Journal of Women in Culture and Society, 14, pp. 856-874.

BOSE Christine E. (1986) « Puerto Rican Women in the United States : An Overview », in Edna Acosta-Belen, Ed., The Puerto Rican Woman : Perspectives on Culture, History, and Society, second edition, 147-169. New York : Praegar.

BOURGOIS Philippe (1995a) In Search of Respect : Selling Crack in El Barrio. New York : Cambridge University Press. Series : Structural Analysis in the Social Sciences.

BOURGOIS Philippe (1995b) « Mothers and Crack », in Nancy Scheper-Hughes and Carolyn Seargant, Eds., Mothers and Children at Risk, Berkeley : University of California Press.

BOURGOIS Philippe (1995c) « The Political Economy of Resistance and Self-Destruction in the Crack Economy : An Ethnographic Perspective ", Annals of the New York Academy of Sciences, Special Issue, « The Anthropology of Lower Income Urban Enclaves : The Case of East Harlem » Ed., Judith Freidenberg.

BURTON Linda (1991) «Caring for Children », The American Enterprise, 2-3, pp. 34-37.

BUTLER Judith and SCOTT Joan Eds. (1992) Feminists Theorize the Political. New York : Routledge.

CAMPBELL Anne (1991) Girls in the Gang, The Second Edition. Cambridge, MA : Basil Blackwell.

CONNELL R.W. (1987) Gender and Power, Stanford, CA : Stanford University Press.

COOK-LYNN Elizabeth (1989) « (Review) The Broken Cord », Wicazo Sa 5 (2), pp. 42-45.

Critique of Anthropology (1993) 13 (3) (Special issue on U.S. inner-city poverty and the « underclass debate ».)

DE LA CANCELA Victor (1986) «A Critical Analysis of Puerto Rican Machismo : Implications for Clinical Practice », Psychotherapy, 23 (2), pp. 291-296.

DIETZ James L. (1986) Economic History of Puerto Rico : Institutional Change and Capitalist Development, Princeton : Princeton University Press.

DORRIS Michael (1989) The Broken Cord: A Family's Ongoing Struggle with Fetal Alcohol Syndrome, New York : Harper \& Rowe.

FELDMAN Allen (1991) Formations of Violence: The Narrative of the Body and Political Terror in Northern Ireland, Chicago : University of Chicago Press.

FERGUSON Brian and FARRAGHER Leslie (1988) The Anthropology of War : A Bibliography, New York: H.F. Guggenheim Foundation.

GIDDENS Anthony (1991) Modernity and Self-Identity : Self and Society in the Late Modern Age, Cambridge : Polity.

GRAMSCI Antonio (1971) Selections from the Prison Notebooks, London : Lawrence and Wishart.

GUARNACCIA Peter J., DE LA CANCELA Victor and CARRILLO Emilio (1989) « The Multiple Meaning of Ataques de Nervios in the Latino Community ", Medical Anthropology, 11 : 47-62.

HUFF C. Ronald, Ed. (1990) Gangs in America. Newbury Park, CA : Sage.

HUNT Robert C. (1971) «Components of Relationships in the Family : A Mexican Village », in Francis L.K. Hsu (dir.), Kinship and Culture, Chicago, Aldine.

JAGGAR Alison (1983) Feminist Politics and Human Nature, Sussex : The Harvester Press. 
JARRET Robin L. (1994, February) « Living Poor : Family Life Among Single Parent, AfricanAmerican Women », Social Problems, 41(1) pp. 30-49.

JEFFERSON, Tony (1994) « Theorising Masculine Subjectivity », in T. Newburn and E. Stanko, Eds., Just Boys Doing Business : Men, Masculinities, and Crime, 10-31, London : Routledge.

KATZ Michael (1986) In the Shadow of the Poorhouse : A Social History of Welfare in America. New York : Basic Books.

LEWIS Oscar (1966) La Vida : A Puerto Rican Family in the Culture of Poverty-San Juan and New York, New York Random House.

LEWIS-FERNANDEZ Robert (1992) « Ataques de Nervios or Panic Attacks : An Embodied Contestation of Puerto Rican Ethnicity », Paper prepared for AAA session, 1 « Healing, Bodily Practices, and Caribbean Ethnicity ", San Francisco, December 2.

MALVEAUX Julianne (1987) « The Political Economy of Black Women », in Mike Davis, Manning Marable, Fred Pfeil, and Michael Sprinker, Eds., The Year Left 2 : An American Socialist Yearbook, 52-73, London : Verso.

MAUER Mark (1992) « Americans Behind Bars : One Year Later », Washington D.C.: The Sentencing Project.

MELENDEZ Edwin and MELENDEZ Edgardo Eds. (1993) Colonial Dilemma : Critical Perspectives on Contemporary Puerto Rico, Boston : South End Press.

MESSERSCHMIDT James W. (1993) Masculinities and Crime : Critique and Reconceptualization of Theory, MD : Rowman \& Littlefield Publishers, Inc.

MOHANTY Chandra Talpade (1984) « Under Western Eyes : Feminist Scholarship and Colonial Discourses », Boundary, 2, 12 (3)/13 (1), pp. 333-358.

The New York Times Magazine (1994, December 4) « Who Will Help the Black Man? A Symposium Moderated by Bob Herbert », pp. 72-77, 90, 92-93, 109-110.

New York Times (1994, March 31) « Sharp Increase Along the Borders of Poverty », p. A8 (Jason DeParle).

PAREDES Americo (1971) « The United States, Mexico, and Machismo ", Journal of the Folklore Institute. 8 (1), pp. 17-37.

PEREZ Sonia and CRUZ Steven (1994, August) Speaking out Loud: Conversations with Young Puerto Rican Men. Washington D.C.: National Council of La Raza.

POLLITT Katha (1990, March 26) « A New Assault on Feminism », The Nation, pp. 408-417.

RAINWATER Lee and YANCEY William Eds. (1967) The Moynihan Report and the Politics of Controversy Boston : MIT Press.

RAMIREZ Rafael (Forthcoming) Dime Capitan : Reflexiones sobre la Masculinidad. San Juan : Huracan Ediciones.

RAPP Rayna (1987) « Urban Kinship in Contemporary America: Families, Classes and Ideology », in Leith Mullings, Ed., Cities of the United States: Studies in Urban Anthropology, 219-243, New York : Columbia University Press.

RIVERA-BATIZ, FRANCISCO L. and SANTIAGO Carlos (1994, April) « The Labor Market and Socioeconomic. Performance of the Puerto Rican Population in the United States 1980-1990 », Monograph prepared for The National Puerto Rican Coalition, Washington D.C. 
RODRIGUEZ Clara E. (1989) Puerto Ricans : Born in the U.S.A. Winchester, MA : Unwin Hyman.

SASSEN Saskia (1991) The Global City : New York, London, Tokyo, Princeton : Princeton University Press.

SINGER Merrill, FREDDIE Valentin, HANS Baer and ZHONGKE Jia (1992) « Why Does Juan Garcia Have a Drinking Problem ? The Perspective of Critical Medical Anthropology », Medical Anthropology, 14, pp. 77-108.

SLUKA Jeffrey A. (1990) « Participant Observation in Violent Social Contexts », Human Organization, 49 (2), pp. 114-126.

STACK Carol (1975) All Our Kin : Strategies for Survival in a Black Community, NY : Harper.

TAUSSIG Michael (1987) Shamanism, Colonialism, and the Wild Man : A Study in Terror and Healing, Chicago : University of Chicago Press.

WACQUANT Loïc (1995) «The New Urban Color Line : The State and Fate of the Ghetto in Postfordist America ", in Craig J. Calhoun, Ed., Social Theory and the Politics of Identity, XX-XX, New York : Basil Blackwell.

\section{NOTES}

1. The New York Times Magazine, 4 décembre 1994.

2. Voir les critiques de Katz (1986) et le numéro spécial de Critique of Anthropology (1993).

3. -On trouvera des recensions des écrits sur les familles et la pauvreté chez Baca Zinn (1989) et Rapp (1987) ; Voir également Malveaux (1987).

4. -Dans les deux lotissements autour de mon immeuble, un peu plus de $70 \%$ des foyers monoparentaux avec enfants en bas âge vivaient en dessous du seuil de pauvreté, en comparaison, le taux était approximativement de $47 \%$ pour l'ensemble des familles d'East Harlem (U.S Census Bureau, census of population and housing, New York Country, By Tract. 1990).

5. -On trouvera une analyse de l'inégale intégration des femmes portoricaines dans la main-d'œuvre américaine chez Bose (1986). Les données du recensement des années 1990 font apparaître une augmentation de la participation des femmes portoricaines dans les années 1980 et le début des années 1990 (Rivera-Batiz and Santiago, 1994). 6. -Voir dans Singer et al. (1992) un compte rendu similaire de l'alcoolisme dans les milieux émigrés portoricains.

7. -Voir Jefferson (1994) pour une présentation théorique de la nécessité d'une interprétation non dualiste de la relation personnalité/société.

8. -Jeu de mots sur [to run a train] : faire rouler un train sur et [to train] : former, (NDT).

9. -Dans son analyse du machisme dans un village mexicain, Hunt note que contrairement au stéréotype suivant lequel les hommes machistes seraient comme poussés à un multipartenariat violent et à l'abus de drogue par de profonds complexes d'infériorité, les « vrais machos » ont une confiance totale en leur domination absolue et peuvent se montrer « extrêmement puritains et souvent profondément impliqués dans ce qu'ils appellent le progrès, l'honnêteté et la justice. Ils sont les exemples plus que parfaits de ce que devrait être le parfait père en fonction de leurs propres normes culturelles : tolérants, sages, justes, honnêtes » (1971 : 116). 
10. -Voir les critiques de l'interprétation de Dorris (1989) du syndrome de l'alcoolisme fotal chez les Indiens d'Amérique comme étant causé par la rupture des rôles féminins traditionnels (Pollitt 1990 ; Cook-Lynn, 1989).

\section{RÉSUMÉS}

Le vécu des hommes portoricains de la seconde génération qui participent à l'économie clandestine des ghettos illustre l'influence de la question du genre sur la souffrance sociale. Cet article s'intéresse à la manière dont les hommes portoricains des ghettos, confinés aux marges d'une nation ouvertement hostile à leur culture et que leur force de travail n'intéresse plus, reconstruisent leur conception de la masculinité par le biais de la violence interpersonnelle, du parasitisme économique et de la domination sexuelle. En nombre croissant, ces hommes désespérés et frustrés se sont réfugiés dans une culture protestataire de rue dont le fondement matériel et l'attrait idéologique s'enracinent dans l'économie de la drogue, alternative concrète à leur exclusion de l'économie licite et de la culture anglo-centrée. Loin d'être les simples jouets de forces structurelles et idéologiques plus vastes, les dealers qui participent à la culture de la rue sont en réalité les agents actifs d'une quête de la dignité - même si cette recherche se manifeste par la violence et l'autodestruction.

Violence, Respect and Sexuality among Puerto Rican Drug Dealers in East Harlem, New York. -The experience of second-generation Puerto Rican men who participate in the underground economy of the ghetto illustrates how gender affects social distress. This article concerns how Puerto Rican men of the ghetto who are stuck on the margins of a society that is no longer interested in their labour power rebuild their notion of masculinity via interpersonal violence, economic parasitism and sexual dominance. A growing number of these desperate and frustrated men have taken refuge in a street culture of protest whose material basis and ideological attraction are rooted in the drug trade, a concrete alternative to their exclusion from the legal economy and an Anglo-centred culture. Far from being the playthings of wider structural and ideological factors, the dealers who participate in street culture are agents actively engaged in a search for dignity -- even when this search takes the form of violence and self-destruction.

Violencia, respeto y sexualidad entre los revendedores de crack puertorriqueños de East Harlem, Nueva York. -- El modo de vivir de los hombres puertorriqueños de le la segunda generación que participan a la economía clandestina de los ghettos ilustra la influencia de la problemática del genero sobre el sufrimiento social. Este articulo estudia la manera en la que esta población esta confinada en el margen de una nación abiertamente hostil a su cultura y donde su fuerza de trabajo ha dejado ya de interesar. En ese contexto el grupo reconstruye su concepción de la masculinidad a través de la violencia interpersonal, del parasitismo económico y de la dominación sexual. Cada vez mas numerosos, estos hombres desesperados y frustrados, van refugiándose en una cultura de protesta urbana donde el fundamento material y la atracción ideológica hunden sus raíces en la economía de la droga como alternativa concreta a la exclusión de la economía formal y a la cultura anglocentrada. Lejos de ser simples marionetas de fuerzas estructurales e ideológicas mas generales, los pequeños traficantes que participan de una cultura de la calle son, en realidad, agentes activos de una búsqueda de dignidad, aun si ella se manifiesta a través de la violencia y de la autodestrucción. 
INDEX

Mots-clés : violence, identité collective, ville, sexualité, toxicomanie, ghetto, sexe, genre, Portoricains, économie informelle

Index géographique : New York

\section{AUTEUR}

\section{PHILIPPE BOURGOIS}

Professor and Chair, Department of Anthropology, History and Social Medicine, University of California, San Francisco. San Francisco, California 94143-0850. 